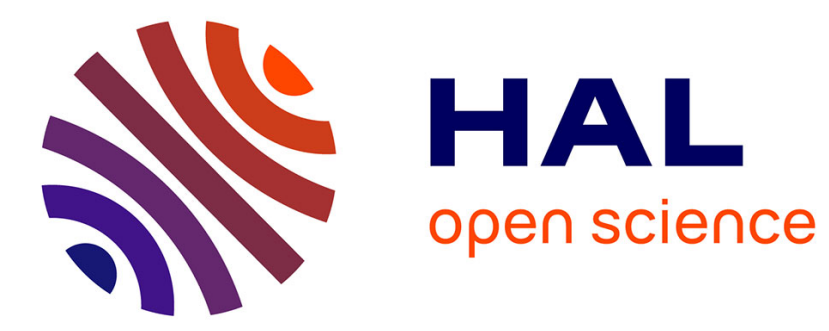

\title{
Heureuses rencontres. Vers le développement d'approches intégrées en géosciences et archéologie \\ Bruno Gavazzi
}

\section{To cite this version:}

Bruno Gavazzi. Heureuses rencontres. Vers le développement d'approches intégrées en géosciences et archéologie. Archimède: archéologie et histoire ancienne, 2020, 7, pp.114-118. 10.47245/archimede.0007.act.01 . halshs-02893346

\section{HAL Id: halshs-02893346 \\ https://shs.hal.science/halshs-02893346}

Submitted on 8 Jul 2020

HAL is a multi-disciplinary open access archive for the deposit and dissemination of scientific research documents, whether they are published or not. The documents may come from teaching and research institutions in France or abroad, or from public or private research centers.
L'archive ouverte pluridisciplinaire HAL, est destinée au dépôt et à la diffusion de documents scientifiques de niveau recherche, publiés ou non, émanant des établissements d'enseignement et de recherche français ou étrangers, des laboratoires publics ou privés. 


\section{ARCHIMÈDE N N 7}

\section{DOSSIER THÉMATIQUE : GESTES RITUELS. DE LA TRACE À L'INTERPRÉTATION}

\section{ACTUALITÉ DE LA RECHERCHE GÉOSCIENCES ET ARCHÉOLOGIE : INTERACTIONS, COMPLÉMENTARITÉS ET PERSPECTIVES}

114 Bruno GAVAZZI

Heureuses rencontres. Vers le développement d'approches intégrées en géosciences et archéologie

119 Jean-Paul BRAVARD

Dialogue interdisciplinaire : de l'unité stratigraphique aux interactions culture-environnement

129 Ferréol SALOMON

Les origines d'Ostie : quelles interactions avec la dynamique d'embouchure ? (Delta du Tibre, Italie)

141 Quentin BORDERIE, Rowena Y. BANERJEA, Stéphane BONNET, Yannick DEVOS, Cristiano NICOSIA, Christophe PETIT, Ferréol SALOMON, Nathalie SCHNEIDER, Barbora WOUTERS, \& Patrice WUSCHER Géoarchéologies des contextes urbains : mieux comprendre les modalités de l'artificialisation des géosystèmes

158 Patrice WUSCHER, Christophe JORDA, Quentin BORDERIE, Nathalie SCHNEIDER \& Laurent BRUXELLES De la formation géologique à la tranchée : trouver et comprendre les sites archéologiques menacés par les travaux d'aménagement du territoire

176 Morgan MILLET \& Michel GUÉLAT

Les vestiges antiques de Rennaz-Noville (Vaud, Suisse) et leur contexte sédimentaire : nouvelles évidences de l'écroulement du Tauredunum

188 Théophile PIAU, François BÉTARD, Fabienne DUGAST, Gilles ARNAUD-FASSETTA \& Vincent VIEL Dynamique géomorphologique holocène et occupation humaine dans le bassin-versant de l'Eure (Bassin de Paris, France) : potentiels d'une approche géoarchéologique multiscalaire et diachronique

205 Dominique SCHWARTZ, Vincent ROBIN, Pierre ADAM, Philippe SCHAEFFER, Anne GEBHARDT, Pierre-Alexis HERRAULT, Benjamin KELLER, Daniele DAPIAGGI, Claire STEVENEL, Maxime THISS, Martine TRAUTMANN \& Damien ERTLEN

Les géosciences au service de l'archéologie agraire. Une étude de cas sur les rideaux de culture de Goldbach (68)

217 Étienne MANTEL, Stéphane DUBOIS, Jonas PARÉTIAS, Victor VISQUESNEL-SCHLOSSER, Corentin VOISIN, Bruno GAVAZZI \& Matthieu RICHARD

Étudier I'occupation d'une ville : les enjeux du PCR « Topographie générale et insertion territoriale de l'agglomération antique de Briga »

231 Charlène MOREL

Un modèle pour comprendre l'influence de l'état de la recherche, des processus post-déposition et de l'attractivité sur la découverte des sites archéologiques dans le Kochersberg (Bas-Rhin, Alsace, Grand-Est, France)

241 Lizzie SCHOLTUS

Spatialisation des découvertes, modélisation du passé. L'informatique au service de I'archéologie

254 Guillaume HULIN \& François-Xavier SIMON

Inrap et géophysique : vers une approche raisonnée

260 Hugo REILLER, Matthieu FUCHS, \& Bruno GAVAZZI

Approche multi-méthodes expérimentale pour l'étude d'un site d'occupation romaine et médiévale à Horbourg-Wihr

272 François-Xavier SIMON, Julien GUILLEMOTEAU, Guillaume HULIN, Joachim RIMPOT, Julien THIESSON \& Alain TABBAGH

De nouvelles perspectives pour les applications des méthodes électromagnétiques basse fréquence en archéologie

283 Rémy WASSONG \& Bruno GAVAZZI

Apport des prospections magnétiques haute résolution à la compréhension d'un habitat protohistorique :

l'exemple du site de hauteur fortifié du Maimont 


\title{
ACTUALITÉ DE LA RECHERCHE \\ « GÉOSCIENCES ET ARCHÉOLOGIE : INTERACTIONS, COMPLÉMENTARITÉS ET PERSPECTIVES » dir. Bruno GAVAZZI
}

\section{HEUREUSES RENCONTRES \\ VERS LE DÉVELOPPEMENT D'APPROCHES INTÉGRÉES EN GÉOSCIENCES ET ARCHÉOLOGIE}

\author{
Bruno GAVAZZI 1, * \\ ${ }^{1}$ Institut de Physique du Globe de Strasbourg (IPGS) ; UMR 7516 ; \\ Université de Strasbourg/EOST, CNRS \\ *bgavazzi@unistra.fr
}

RÉSUMÉ

Ce dossier «Géosciences et archéologie : interactions, complémentarités et perspectives » rassemble quatorze études qui s'inscrivent dans une même démarche : réunir différentes communautés scientifiques et professionnelles autour de sujets communs pour répondre à des questionnements complexes et faire émerger de nouvelles compétences et de nouvelles approches à la frontière de nos disciplines. Les contributions ici réunies constituent des points d'étape d'une démarche fondamentalement interdisciplinaire : il s'agit, de façon collective, d'ouvrir de nouvelles voies pour mieux comprendre le passé humain et environnemental ainsi que l'évolution de leurs interactions jusqu'au périodes actuelles. Les résultats des premières « Rencontres interdisciplinaires et interprofessionnelles Géosciences - Archéologie » sont très prometteurs et soulèvent l'importance d'une recherche scientifique prête à remettre en question ses frontières traditionnelles. Le résultat d'un croisement de

\section{MOTS-CLÉS}

Interdisciplinarité, multiprofessionalisme, socioécosystèmes, géoarchéologie, géophysique, archéométrie. compétences offre davantage que la simple somme de celles-ci et ce dossier en est l'expression : il illustre la qualité de la recherche en géosciences et en archéologie comme de la dynamique de ses actrices et acteurs.
This dossier « Géosciences et archéologie : interactions, complémentarités et perspectives » is constituted by fourteen original articles which follow a common approach: bringing together different scientific and professional communities around common subjects to answer complex questions and to bring out new skills and approaches at the frontiers of our disciplines. The papers gathered here are milestones of a fundamentally interdisciplinary approach: the aim is to open collectively new ways for a better understanding of the records of the human and environmental past as well as their current evolutions. The results of the first « Rencontres interdisciplinaires et interprofessionnelles Géosciences - Archéologie » are therefore very promising and show the significance of a scientific research that is ready to question its traditional habits and boundaries. Crossing different skills offers more than their simple addition. This dossier illustrates this fact as well as the quality of research in geosciences and archaeology and the dynamics of the contributors.
KeYwords

Interdisciplinarity, multiprofessionalism, socioecosystems, geoarchaeology, geophysics, archaeometry. 
L'apport des géosciences [1] pour répondre à des problématiques archéologiques est aujourd'hui pleinement reconnu, si bien que leur utilisation apparaît de plus en plus fréquente, que ce soit en contexte d'études programmées ou préventives. Ce dynamisme peut se traduire, entre autres, par le développement de l'archéométrie, qui consiste en la mise en œuvre de méthodes physiques ou chimiques pour l'interprétation et la datation en archéologie, et de la géoarchéologie, à savoir l'étude de l'évolution des environnements en relation avec les peuplements. Si la pluridisciplinarité de ces démarches ne fait aucun doute, il apparaît que la relation entre géosciences et sciences historiques reste majoritairement une relation outils/applications sur des cas précis avec pour objectif la compréhension du passé. Pourtant, nos interactions génèrent des découvertes et des innovations nouvelles dans nos mondes respectifs, et elles conduisent à développer de nouvelles approches, le plus souvent dans l'objectif d'une adaptation à des contextes particuliers où certaines méthodes s'avèrent plus efficaces que d'autres. Ce dossier scientifique intitulé «Géosciences et archéologie : interactions, complémentarités et perspectives » s'inscrit alors dans une démarche plus large qui vise à fédérer des communautés scientifiques et professionnelles diverses autour de sujets communs et à répondre aux questionnements suivants : comment travaillons-nous ensemble ? De quelle manière sommes-nous complémentaires et comment l'être encore davantage? Et dans quelles directions voulons-nous aller pour, en reprenant la devise du CNRS, dépasser les frontières?

[1] Les géosciences sont entendues ici au sens large, c'est-à-dire à tout ce qui touche à la Terre, ce qui englobe de nombreuses disciplines, comme par exemple la géologie, la géochimie, la géographie, la géophysique, etc.

[2] Les IdEx ou « Initiatives d'excellence » sont un mode de financement par appel à projet financé par l'État dans le cadre des investissements d'avenir. L'Université de Strasbourg fait partie dès 2010 des trois lauréats initiaux de ces IdEx, ce qui lui permet d'organiser chaque année des appels à projets sur différentes thématiques. Plus concrètement, ce colloque s'inscrit dans I'IdEx « Manifestations et initiatives à destination du monde socio-économique » de l'année 2018 et a reçu un financement à ce titre.
Cette démarche est tout d'abord née d'un constat qui s'est construit sur plusieurs années à travers de multiples collaborations entre différents mondes, celui de la recherche académique, celui de la culture et du patrimoine, ainsi que celui de l'aménagement du territoire, voire celui du monde industriel et des développeurs d'équipements (imagerie de la proche surface et analyses) et de solutions informatiques de traitement des données. Il existe à la frontière des disciplines et professions concernées des objets d'études communs, des approches et des méthodes partagées et des expertises complémentaires qui sont aujourd'hui peu explorées. Ainsi, nous avons fait le pari de réunir différentes communautés à la fois disciplinaires - rattachées à des Collégiums universitaires et des instituts du CNRS différents -, mais aussi professionnelles, aux cultures, coutumes et fonctionnements différents pour ouvrir de nouvelles voies scientifiques et applicatives communes. La première étape de cette démarche a été la tenue des premières Rencontres interdisciplinaires et interprofessionnelles Géosciences - Archéologie qui ont eu lieu à I'Université de Strasbourg les 17 et 18 juin 2019. L'intérêt de communautés diverses et l'adhésion à la démarche proposée peut s'illustrer en premier lieu par l'implication de différentes institutions. L'événement a pu réunir le soutien de l'IdEx [2] de I'Université de Strasbourg, du CNRS [3], du ministère de la culture [4] et de la ville de Strasbourg. De plus, différents acteurs ont participé à son organisation : trois unités mixtes de recherche (Unistra/ CNRS) (sciences de la Terre [5], histoire et archéologie [6], et géographie [7]), Archéologie Alsace, I'Institut national de recherches archéologiques

[3] Ce soutien s'est traduit par un financement de I'Institut national des sciences de I'Univers (INSU).

[4] Ce soutien s'est traduit par un financement de la Direction régionale des affaires culturelles (DRAC) du Grand Est.

[5] CNRS/UNISTRA UMR 7516 IPGS - Institut de Physique du Globe de Strasbourg.

[6] CNRS/UNISTRA UMR 7044 Archimède - Archéologie et Histoire ancienne : Méditerranée - Europe.

[7] CNRS/UNISTRA UMR 7362 LIVE - Laboratoire Image, Ville, Environnement. 
préventives (Inrap) et l'association professionnelle Groupe des Méthodes Pluridisciplinaires Contribuant à I'Archéologie (GMPCA). Cet aspect inter communautés se retrouve également dans la diversité des origines des participants, provenant des milieux académiques comme socio-économiques, d'établissements publics comme privés, impliquant différents métiers (chercheurs, enseignants-chercheurs, ingénieurs, techniciens) mais également des étudiants de master et de jeunes collègues préparant un doctorat ou en post-doctorat. Ainsi, ce sont 103 participants provenant de toute la France, mais également d'Algérie, de Grèce, du Maroc et de Suisse, qui ont pu se retrouver autour de trente-trois présentations orales et seize posters, organisés en trois sessions. La première, «Géoarchéologie : à la frontière des disciplines $»$, abordait la théorie, les méthodes et les applications aux frontières entre les sciences de la Terre et de l'archéologie, comprenant par exemple les études des matériaux, les datations radiométriques, la micromorphologie, etc. La deuxième, «Projets interdisciplinaires : une démarche à fort potentiel », visait à regrouper des retours d'expérience sur différents projets interdisciplinaires à succès dans une logique de bilan et exploration des perspectives d'avenir. Enfin, la troisième, « Géophysique et archéologie : de l'imagerie à la caractérisation de l'invisible », concernait le domaine de la géophysique appliquée en archéologie : échanges sur les bonnes pratiques, possibilités actuelles et nouvelles approches innovantes. Des plateformes de services hébergées dans les laboratoires organisateurs ont également été présentées dans le cadre des journées, afin d'informer sur les différents moyens mobilisables par les différentes communautés [8]. Enfin, les rencontres se sont conclues par une table ronde sur les besoins, attentes, moyens et sur l'avenir de la géophysique en archéologie : il s'en est dégagé une volonté forte de développement plus poussé de collaborations entre disciplines et milieux professionnels, encore trop cloisonnés. Se sont trouvés formulés également le besoin de nouvelles compétences interdisciplinaires à l'interface des communautés et la nécessité de nouvelles structures pour conduire leur émergence.

Le dossier présenté ici s'inscrit à la suite de ces rencontres et du succès qu'elles ont rencontré, dans une logique de valorisation et d'approfondissement des thématiques qui s'en sont dégagées. L'objectif est de renforcer et de poursuivre la démarche initiée, dans l'objectif de présenter un état actuel, bien entendu non exhaustif, à travers la présentation de différentes études et approches. Ce dossier débute par une rétrospective sur le dialogue interdisciplinaire ayant contribué à l'établissement de la discipline géoarchéologique [9], puis se poursuit par une étude des origines d'Ostie d'un point de vue géoarchéologique [10]. L'article suivant propose de nouvelles approches géoarchéologiques en contextes urbains [11], puis sont ensuite présentés l'apport et les perspectives de la démarche associant géomorphologues et archéologues en contexte d'archéologie préventive [12]. Les contributions qui suivent concernent des études de cas interdisciplinaires : sur des traces de l'écroulement du Tauredunum en Suisse [13], sur l'occupation et l'évolution du bassin de l'Eure durant l'Holocène [14], sur les rideaux de culture de Goldbach (68) [15] et sur l'agglomération antique de Briga [16]. Ensuite sont présentés deux articles de modélisation pour l'analyse spatiale et statistique : pour mieux comprendre les facteurs de la répartition des sites dans le Kochersberg (Alsace) [17], et pour mettre en évidence et cartographier des groupes régionaux dans l'Est de la Gaule durant l'âge du Fer [18]. Enfin, les quatre dernières publications s'articulent plus particulièrement autour de la thématique des interactions entre la géophysique et l'archéologie : la première fait un état des lieux de l'utilisation de la géophysique à
[8] Ont été présenté ArkeoGIS, plateforme informatique pour la mise en commun et l'analyse de données géoréférencées concernant le passé, Le Laboratoire d'analyse des sols et formations superficielles (LAS), qui réalise des analyses sédimentologiques de la prise d'échantillon à la diffusion des résultats, Le pôle analytique du LhyGes qui réalise des analyses chimiques élémentaires, analyses isotopiques, ainsi que minéralogiques, des plateformes de $\mu \mathrm{XRF}$ et de lames minces, la plateforme Carma qui propose mesures, traitements et interprétations de données magnétiques au sol et aéroportées, et enfin Terremys, startup spécialisée dans la mise au point de solution de mesures magnétiques par drone en collaboration avec le CNRS.
[9] BRAVARD 2020.

[10] SALOMON 2020.

[11] BORDERIE et al. 2020.

[12] Wuscher et al. 2020.

[13] Millet \& GuÉLAT 2020.

[14] Piau et al. 2020.

[15] SchWARTZ et al. 2020.

[16] MANTEL et al. 2020.

[17] MOREL 2020.

[18] SCHOLTUS 2020. 
I'Inrap [19], la deuxième présente une approche multi-méthode dans le cadre d'une superposition complexe de vestiges [20], la troisième fait le point sur l'utilisation et les perspectives des méthodes électro-magnétiques [21] tandis que la dernière montre l'apport d'une méthode magnétique haute résolution pour l'étude de sites de hauteur [22]. Tous ces articles bien que traitant de sujets bien différents ont pour point commun majeur une interdisciplinarité forte entre les sciences dites dures et les sciences dites complexes. Ce dossier démontre, si besoin en était, la pertinence de ces premières rencontres « Géosciences et archéologie », et laisse présager un renforcement de la démarche pluridisciplinaire et le développement de nouvelles collaborations et de nouvelles approches. Ceci est d'autant plus vrai qu'on assiste ces dernières années à un fort décloisonnement des disciplines et à une approche plus systémique.

Ces rencontres et ce dossier posent les premiers jalons de nouvelles approches en pleine expansion, et appellent à l'organisation de futures rencontres et autres événements pour les développer.
Les contributions ici réunies constituent des points d'étape d'une démarche fondamentalement interdisciplinaire : il s'agit, de façon collective, d'ouvrir de nouvelles voies pour mieux comprendre le passé humain et environnemental ainsi que les évolutions de leurs interactions jusqu'au périodes actuelles. Au-delà, ces aspects s'inscrivent dans la perspective d'une meilleure compréhension des socio-écosystèmes complexes. Cette compréhension est essentielle pour répondre aux crises actuelles du changement de la biodiversité et du climat qui occupent une place croissante dans les préoccupations sociétales. En outre, ces enjeux et ce contexte soulèvent l'importance d'une recherche scientifique prête à remettre en question ses frontières traditionnelles.

[19] HULIN \& SimON 2020.

[20] Reiller, Fuchs \& Gavazzi, 2020.

[21] Simon et al. 2020.

[22] WASSONG \& GAVAZZI 2020.

\section{REMERCIEMENTS}

Je souhaite remercier en premier lieu tous les acteurs ayant participé au financement des rencontres et de ce dossier : I'Université de Strasbourg, le CNRS (INSU), Archéologie Alsace et du Ministère de la Culture et de la Communication (DRAC Grand Est, dont notamment Nicolas Payraud). Je tiens à remercier tout particulièrement la ville de Strasbourg pour son soutien et l'organisation d'une réception à l'hôtel de ville qui fut un lieu d'échange convivial pour tous les participants. Merci également au bureau du GMPCA pour son soutien et son action de diffusion. Je remercie les membres du comité d'organisation que j'ai eu I'honneur de présider: Frédéric Colin (Archimède), Michel Humm (Archimède), Guillaume Hulin (Inrap), Laurence Jouniaux (IPGS), Philippe Kuchler (Archéologie Alsace), Ferréol Salomon (LIVE) et Mathieu Schuster (IPGS), ainsi que tous les membres de la cellule congrès du service de formation continue de l'Unistra qui ont été d'une aide précieuse pour toute la logistique. Merci au comité de rédaction de la revue Archimède qui a soutenu pleinement cette démarche de publication, et tout particulièrement à Loup Bernard, Sandra Boehringer et Marie-José Morant pour leur aide précieuse au processus d'édition du dossier. Enfin, un grand merci aux participants des rencontres, aux 50 auteur.e.s des différentes contributions de ce dossier et aux 25 rapporteur.e.s les ayant évaluées.

\section{BIBLIOGRAPHIE}

Borderie, Quentin, Banerjea, Rowena Y., Bonnet, Stéphane, Devos, Yannick, Nicosia, Cristiano, Petit, Christophe, Salomon, Ferréol, Schneider, Nathalie, Wouters, Barbora \& Wuscher, Patrice, 2020, « Géoarchéologies des contextes urbains : mieux comprendre les modalités de l'artificialisation des géosystèmes 》, Archimède. Archéologie et histoire ancienne 7, p. 141-157.

BRAVARD, Jean-Paul, 2020, « Dialogue interdisciplinaire : de I'unité stratigraphique aux interactions culture-environnement », Archimède. Archéologie et histoire ancienne 7, p. 119-128. 
Hulin, Guillaume \& Simon, François-Xavier, 2020, « Inrap et géophysique : vers une approche raisonnée », Archimède. Archéologie et histoire ancienne 7, p. 254-259.

Mantel, Étienne, Dubois, Stéphane, Parétias, Jonas, Visquesnel-Schlosser, Victor, Voisin, Corentin, GavazzI, Bruno \& RICHARD, Matthieu, 2020, «Étudier l'occupation d'une ville : les enjeux du PCR «Topographie générale et insertion territoriale de l'agglomération antique de Briga» », Archimède. Archéologie et histoire ancienne 7 , p. 217-230.

Millet, Morgan \& GuÉLAT, Michel, 2020, « Les vestiges antiques de Rennaz-Noville (Vaud, Suisse) et leur contexte sédimentaire : nouvelles évidences de l'écroulement du Tauredunum », Archimède. Archéologie et histoire ancienne 7, p. $176-187$.

MOREL, Charlène, 2020, « Un modèle pour comprendre l'influence de l'état de la recherche, des processus taphonomiques et de l'attractivité sur la découverte des sites archéologiques dans le Kochersberg (Bas-Rhin, Alsace, Grand-Est, France) 》, Archimède. Archéologie et histoire ancienne 7, p. 231-240.

Piau, Théophile, Bétard, François, Dugast, Fabienne, Arnaud-Fassetta, Gilles \& Viel, Vincent, 2020, «Dynamique géomorphologique holocène et occupation humaine dans le bassin-versant de l'Eure (Bassin de Paris, France) : potentiels d'une approche géoarchéologique multiscalaire et diachronique », Archimède. Archéologie et histoire ancienne 7, p. 188-204.

Reiller, Hugo, Fuchs, Matthieu \& GavazzI, Bruno, 2020, « Approche multi-méthodes expérimentale pour l'étude d'un site d'occupation romaine et médiévale à Horbourg-Wihr », Archimède. Archéologie et histoire ancienne 7 , p. 260-271.

SALOMON, Ferréol, 2020, « Les origines d'Ostie : quelles interactions avec la dynamique d'embouchure ? (Delta du Tibre, Italie) », Archimède. Archéologie et histoire ancienne 7, p. 129-140.

Scholtus, Lizzie, 2020, «Spatialisation des découvertes, modélisation du passé. L'informatique au service de l'archéologie », Archimède. Archéologie et histoire ancienne 7, p. 241-253.

Schwartz, Dominique, Robin, Vincent, Adam, Pierre, Schaeffer, Philippe, Gebhardt, Anne, Herrault, Pierre-Alexis, Keller, Benjamin, Dapiaggr, Daniele, Stevenel, Claire, Thiss, Maxime, Trautmann, Martine \& ERTLEN, Damien, 2020, « Les géosciences au service de l'archéologie agraire. Une étude de cas sur les rideaux de culture de Goldbach (68) », Archimède. Archéologie et histoire ancienne 7, p. 205-216.

Simon, François-Xavier, Guillemoteau, Julien, Hulin, Guillaume, Rimpot, Joachim, Thresson, Julien \& TABBAGH, Alain, 2020, «De nouvelles perspectives pour les applications des méthodes électromagnétiques basse fréquence en archéologie », Archimède. Archéologie et histoire ancienne 7, p. 272-282.

WASSONG, Rémy \& GAVAzzI, Bruno, 2020, « Apport des prospections magnétiques haute résolution à la compréhension d'un habitat protohistorique : l'exemple du site de hauteur fortifié du Maimont », Archimède. Archéologie et histoire ancienne 7, p. 283-293.

Wuscher, Patrice, Jorda, Christohe, Borderie, Quentin, Schneider, Nathalie \& Bruxelles, Laurent, 2020, «De la formation géologique à la tranchée : trouver et comprendre les sites archéologiques menacés par les travaux d'aménagement du territoire », Archimède. Archéologie et histoire ancienne 7, p. 158-175. 